Mots. Les langages du politique

Présidentielle 2007. Scènes de genre

\title{
Questions de citoyens, questions de genre
}

Pierre-Olivier Dupuy et Pascal Marchand

\section{(2) OpenEdition}

Journals

Édition électronique

URL : https://journals.openedition.org/mots/19083

DOI : $10.4000 /$ mots. 19083

ISSN : 1960-6001

\section{Éditeur}

ENS Éditions

\section{Édition imprimée}

Date de publication : 1 juillet 2009

Pagination : 83-99

ISBN : 978-2-84788-151-6

ISSN : 0243-6450

\section{Référence électronique}

Pierre-Olivier Dupuy et Pascal Marchand, "Questions de citoyens, questions de genre », Mots. Les langages du politique [En ligne], 90 | 2009, mis en ligne le 01 juillet 2011, consulté le 22 avril 2022. URL : http://journals.openedition.org/mots/19083; DOI : https://doi.org/10.4000/mots.19083 


\section{Pierre-Olivier Dupuy, Pascal Marchand}

\section{Questions de citoyens, questions de genre}

L'élection présidentielle de 2007 a vu apparaitre de nouvelles émissions politiques dans lesquelles la parole journalistique laisse place à la parole citoyenne. C'est le cas de «J'ai une question à vous poser» (TF1) dans laquelle un panel de Français, constitué par un institut de sondage, interroge directement les candidats. Un tel concept avait déjà été expérimenté, le 14 avril 2005, lors d'un débat entre Jacques Chirac et quatre-vingt-trois jeunes avant le référendum sur le Traité constitutionnel européen. Cependant, la parole profane n'avait ni l'exclusivité du débat - quatre animateurs interrogeaient également le Président - ni une réelle liberté d'expression: la parole citoyenne apparaissait encore largement «domestiquée» (Neveu, 1995, p. 153) par l'appareil médiatico-journalistique (faible pouvoir de relance, reformulation de la question par l'un des animateurs, etc.). Si les conditions de prise de parole restent relativement floues dans l'émission «l'ai une question à vous poser» - Patrick Poivre d'Arvor affirme sur un chat n'avoir opéré aucune sélection parmi les questions posées et n'avoir procédé qu'à des regroupements thématiques ${ }^{1}$-, l'animateur s'est effacé au profit de la parole profane. Certes, la centaine d'invités présents n'ont pas pu tous s'exprimer. En revanche, ceux qui ont pris la parole ont pu interagir en bénéficiant d'un pouvoir de relance permettant la remise en cause des réponses apportées par les candidats. En ce sens, l'émission de TF1 se rapproche du modèle interactif des échanges politiques (Mouchon, 1995) : la relation directe et plus égalitaire entre citoyens et personnel politique. Ce regain égalitaire se traduit, selon nous, par une modification du statut des citoyens. Le profane n'est plus uniquement considéré comme témoin du monde, interrogé pour son vécu (Darras, 1994; Mouchon, 2001; Charaudeau, 2005), mais également invité comme acteur légitime de l'espace public. En acceptant de se confronter à la parole profane devant des millions de téléspectateurs² ${ }^{2}$ les personnalités politiques mettent en jeu leur

1. Chat du 28 février 2007 réalisé sur LCI.fr [URL: http://tf1.lci.fr/infos/elections-2007/question_ poser/], site consulté le 4 mars 2009.

2. Les émissions politiques de la campagne 2007 ont réalisé des audiences honorables (source: Médiamétrie) et ce, malgré le déclin des émissions politiques (Neveu, 1995; Mouchon, 2001). 
image (c'est-à-dire leur «construit social identitaire»: Moliner, 1996; Deschamps et Moliner, 2008), bien davantage que dans un rapport classique - et, somme toute, plus attendu - entre le journaliste et l'homme politique. Même si les invités politiques maitrisent mieux que les citoyens les conditions de la prise de parole télévisée (Darras, 1994), ils sont ici confrontés aux préoccupations des simples citoyens qui leur reprochent d'être déconnectés de la vie quotidienne (Mouchon, 1995, 2001). Ainsi, au lendemain de sa prestation télévisée, José Luis Zapatero3 suscitait la polémique suite à une question sur le prix d'un café dont il ne connaissait pas la valeur. Si ces questions pécuniaires renvoient à un vécu, les profanes n'en sont pas moins capables d'élaborer des inférences plus générales à partir de ce même vécus: «For example, people do not need the mass media to alert them to many aspects of the economy. Personal experience usually informs people about pricing patterns at Christmas or about rising gas prices. These are obtrusive features of the economy.» (McCombs, Reynolds, 2002, p. 8)

L'image d'un candidat est le produit d'un processus de co-construction (Deschamps, Moliner, 2008) qui implique des inférences de part et d'autre: les représentations que l'homme politique se fait de l'opinion - si tant est qu'il existe une seule "opinion publique » (Charaudeau, 2005) - et celles que l'opinion se fait de l'homme politique. Mais s'il est courant d'entendre dire que les personnalités politiques «travaillent leur image», rien ne permet d'affirmer que cette image, donnée à voir, sera correctement interprétée par les citoyens. Dès lors, les questions des citoyens véhiculent des représentations qui peuvent ne pas correspondre à ce que le candidat cherche à montrer de lui-même durant la campagne.

La désaffection des programmes politiques a fait l'objet de nombreuses études: ce n'est pas le journalisme politique qui aurait changé, mais il y aurait un écart grandissant entre un certain idéal de la couverture médiatique de l'actualité politique et les formes actuelles du journalisme politique, c'est-à-dire un fossé entre l'offre médiatique et la demande des citoyens (Nadeau, Giasson, 2003). À côté des émissions politiques traditionnelles, les personnalités politiques peuvent rechercher la fréquentation d'émissions dont le cadre, le ton et le contenu apparaissent comme moins formels, rigides et solennels. Ce fut le cas de «Questions à domicile» (diffusée sur TF1 de 1986 à 1989), qui cherchait à se démarquer par une approche plus intimiste de l'homme politique (Le Grignou, Neveu, 1997). Plus récemment, les émissions d’infotainment ont marqué une évolution notable: la politique s'intègre au divertissement dans une volonté de se rapprocher des «citoyens d'en bas» (Pino, Marchand, 2007).

3. Émission «Tengo una pregunta para usted» (format espagnol de «)'ai une question à vous poser»), diffusée le 27 mars 2007 surTVE. 
Dans cette perspective, nous nous sommes attachés à analyser les questions posées par les citoyens. Bien qu'ayant des compétences sociales et d'élocution différentes, les profanes interrogent sur un même plateau, et dans un laps de temps réduit, un candidat ou une candidate sur des sujets qu'ils ont (normalement) eux-mêmes choisis. Analysé comme une interaction, tout questionnement implique un jeu d'attentes et d'images réciproques qui pèse sur le choix des mots et des formules. Mais la parole citoyenne est-elle à même d'éviter les filtrages journalistiques qui conduisent fréquemment à adapter les questions à leurs cibles (Marchand, 2004) ? Les citoyens reproduisent-ils des biais de discrimination dans leur perception et interrogation du corps politique ou en génèrent-ils d'autres ? À partir de quelles caractéristiques (politiques, physiques, etc.) les citoyens se construisent-ils une image des candidats? Plusieurs hypothèses peuvent être posées, qui font intervenir les caractéristiques des cibles des questions (sexe, parti politique, etc.), mais également celles des sources. Plus précisément: ya-t-il une distinction opérée entre les personnalités de gauche et celles de droite? Entre les petits et les grands candidats (majorité / minorités parlementaires)4 ? Y a-t-il des thématiques et/ou des formulations propres aux genres qui discriminent les hommes et les femmes? Une attention particulière est portée à cette dernière hypothèse : l'élection présidentielle de 2007 ayant, pour la première fois durant la Cinquième République, opposé - au second tour - un homme et une femme, nous nous demandons si la catégorisation sexuelle a pu discriminer les candidates. Nous nous référons ici à la division sexuelle de la société et aux représentations de genre qu'elle génère (Bourdieu, 1998; Ballmer-Cao, Mottier, Sgier, 2000 ; Achin, Lévêque, 2006).

\section{Méthode}

L'approche privilégiée relève de l'analyse statistique des données textuelles (Lebart et Salem, 1994).

\section{Corpus}

Nous avons transcrit les questions des citoyens dans les programmes «J'ai une question à vous poser» (TF1) et «À vous de Juger» (France 2). Dans son dispositif, le programme de France 2 se rapproche de l'émission politique traditionnelle, puisque seule une partie de l'émission est consacrée à la parole citoyenne5. Pourtant, il nous a paru judicieux de l'analyser car ces émissions

4. La distinction petits et grands candidats repose sur une partition du corps politique tel qu'il est conçu dans notre société: deux partis majeurs d'un côté et des partis alternatifs de l'autre.

5. Contrairement à TF1 qui choisit de constituer un échantillon représentatif, France 2 sélectionne 
ont été diffusées pendant la même période, mais aussi parce que la journaliste a tenté d'instaurer, entre l'invité et le citoyen, un dialogue qui dépasse le simple cadre du modèle "question unique-réponse». Le corpus se compose de huit émissions qui correspondent aux quatre principaux présidentiables: Nicolas Sarkozy et Ségolène Royal bénéficient chacun d'une émission entière ; François Bayrou et Jean-Marie Le Pen d'une demi-émission. Le reste des émissions est partagé par les petits candidats:

- TF1: Nicolas Sarkozy (5 février) /Jean-Marie Le Pen puis, invités séparément, Marie-George Buffet, Philippe de Villiers et Olivier Besancenot (12 février) ; Ségolène Royal (19 février) / François Bayrou puis, séparément, Dominique Voynet, José Bové et Arlette Laguiller (26 février);

- France 2:Jean-Marie Le Pen puis, réunis simultanément, DominiqueVoynet, Olivier Besancenot et Nicolas Dupont-Aignan (8 février) / François Bayrou puis, simultanément, Marie-George Buffet, José Bové et Philippe de Villiers (15 février) / Nicolas Sarkozy (8 mars) / Ségolène Royal (15 mars).

Nous avons conservé, lors de la transcription, les particules discursives (bon, voilà, etc.) et les hésitations telles que les phrases inachevées. La transcription correspond donc à ce qui a été dit et entendu lors des émissions.

\section{Variables}

Pour l'analyse, dix variables ont été définies pour décrire chaque question. Elles concernent le sexe des interlocuteurs (masculin/féminin), le nom du candidat interrogé, son appartenance politique 6 , le statut de cette appartenance (distinction entre candidats des principales formations et candidats des formations marginales), la chaine de diffusion. Certaines variables, enfin, croisent deux des variables précédentes (candidat et chaine de diffusion; sexe du citoyen et nom du candidat; etc.).

\section{Analyse statistique du discours}

Nos résultats reposent sur une analyse lexicométrique du corpus réalisée à partir des logiciels Alceste et Lexic03. Un tableau lexical est construit, croisant l'ensemble des formes lexicales du corpus et, tantôt l'ensemble des variables utilisées (Lexico3), tantôt le corpus segmenté en unités de contexte (Alceste), unités qui peuvent correspondre à une ou plusieurs phrases. La classification hiérarchique descendante (Alceste) est une procédure qui permet de définir

elle-même ses citoyens selon des critères qui n'ont pas été communiqués. Les animateurs des deux émissions se défendent d'avoir opéré une sélection parmi les questions que les citoyens voulaient poser.

6. Concernant l'appartenance politique de François Bayrou, le candidat centriste a été catégorisé à droite, son parti ayant souvent conclu, par le passé, des alliances avec la droite. 
des classes statistiquement homogènes à partir du vocabulaire (Reinert, 1990). Pour éviter des artefacts, notamment de genre grammatical, les formes lexicales sont réduites à leur racine pour les substantifs (notée racine+)7 et à l'infinitif pour les verbes (noté racine+er). Quant au calcul des spécificités (Lexico3), il s'agit d'observer la distribution des formes lexicales selon les différentes partitions correspondant aux variables décrites plus haut: plus la distribution d'une forme lexicale se distingue d'une distribution aléatoire, plus cette forme est dite spécifique de la modalité de variable étudiée.

\section{Résultats}

\section{Corpus}

Le corpus compte 278 questions, les trois-quarts provenant de l'émission de TF1 (207 questions). 115 questions ont été posées par des femmes et 163 par des hommes. Les questions se répartissent comme suit.

\begin{tabular}{|c|c|c|c|c|}
\hline & $F^{*}$ & $M^{*}$ & Total & Fréquence \\
\hline Ségolène Royal & 24 & 40 & 64 & $23,0 \%$ \\
\hline Nicolas Sarkozy & 27 & 25 & 52 & $18,7 \%$ \\
\hline François Bayrou & 13 & 31 & 44 & $15,8 \%$ \\
\hline Jean-Marie Le Pen & 14 & 24 & 38 & $13,7 \%$ \\
\hline Marie-George Buffet & 8 & 9 & 17 & $6,1 \%$ \\
\hline Arlette Laguiller & 7 & 10 & 17 & $6,1 \%$ \\
\hline Olivier Besancenot & 4 & 7 & 11 & $4,0 \%$ \\
\hline José Bové & 6 & 4 & 10 & $3,6 \%$ \\
\hline Philippe de Villiers & 4 & 5 & 9 & $3,2 \%$ \\
\hline Dominique Voynet & 3 & 5 & 8 & $2,9 \%$ \\
\hline $\begin{array}{l}\text { Dominique Voynet, Olivier Besancenot, } \\
\text { Nicolas Dupont-Aignan }\end{array}$ & 4 & 3 & 7 & $2,5 \%$ \\
\hline $\begin{array}{l}\text { Marie-George Buffet, José Bové, } \\
\text { Philippe de Villiers }\end{array}$ & 1 & 0 & 1 & $0,4 \%$ \\
\hline Total & 115 & 163 & 278 & $100 \%$ \\
\hline
\end{tabular}

Tableau 1. Nombre de questions posées selon le candidat et le sexe du questionneur

* $\mathrm{F}$ : Sexe de l'intervenant $=$ féminin $; \mathrm{M}$ : Sexe de l'intervenant $=$ masculin.

Le corpus compte 38822 occurrences qui représentent 4398 formes distinctes. La classification hiérarchique nous permet de distinguer, à partir des 839 unités de contextes, deux grands profils lexicaux: la classe $n^{\circ} 1(36,59 \%$ du corpus) et la classe $n^{\circ} 2(63,41 \%$ du corpus).

7. Par exemple, les formes président et présidente sont regroupées sous la même forme président+. 


\section{Des références financières et professionnelles (classe $n^{\circ} 1$ )}

Nombreuses sont les références à l'argent, qu'il s'agisse de rentrées ou de dépenses financières (euro+, salaire+, smic, augment+er, pa+yer, argent, allocat+ion, aide+, loyer+, retraite+, achat+, cotis+er, bénéfici+er, compt+er, gagn+er, financter, charge+) et à l'univers professionnel (salariét, travail, embauch+er, entreprise+, main+, emploit, cdi, heure+, œuvre, anpe, patron, chômeur).

\begin{tabular}{l|c} 
Forme & KHI2 \\
\hline${ }^{*}$ S2=ff & 64,05 \\
${ }^{*}$ sexcan=fvobedu & 33,74 \\
${ }^{*}$ can=vobedu & 27,95 \\
${ }^{*}$ canm=vobedufr2 & 27,95 \\
${ }^{*}$ cansex=f & 27,10 \\
${ }^{*}$ sexcan=froyal & 26,96 \\
${ }^{*}$ m=fr2 & 25,07 \\
${ }^{*}$ Sexcan=fbuffet & 24,12 \\
${ }^{*}$ sex=f & 22,51 \\
${ }^{*}$ canm=buffettf1 & 22,08 \\
${ }^{*}$ can=buffet & 19,85 \\
${ }^{*}$ pol=nil & 17,22 \\
*cansex=nil & 17,22 \\
*s2=f & 16,61 \\
${ }^{*}$ canm=sarkozyfr2 & 14,64 \\
* pol=g & 10,71
\end{tabular}

Tableau 2. Profil lexical de la classe $n^{\circ} 1$ (par Khi2 décroissant) 8

Pour déterminer les facteurs qui expliquent ce lexique, nous calculons une corrélation entre la classe et les modalités de variables codées dans le corpus. Le facteur principal de la classe 1 est une modalité croisant le sexe des deux interlocuteurs (s2=ff): il s'agit surtout d'échanges femme-femme. Dans une moindre mesure, d'autres modalités sont corrélées avec ce lexique : le candidat (can=vobedu)', le sexe du candidat (cansex=f), le sexe de l'intervenant ( $\operatorname{sex}=f$ ) et le sexe de l'intervenant croisé avec le candidat (sexcan=fvobedu; sexcan=froyal; sexcan=fbuffet). Les segments répétés caractéristiques de cette première classe (j'ai, je suis, on est, pouvoir + achat) montrent qu'il s'agit souvent pour les femmes de raconter leur situation personnelle et les difficultés financières qu'elles rencontrent. Les formes euro+ et mois renvoient

8. Nous n'avons reproduit ici que les quarante premières formes réduites.

9. La modalité « vobedu » correspond à l'interaction Voynet / Besancenot / Dupont-Aignan. 


\begin{tabular}{l|r|l|l} 
Forme & $\mathrm{KHI} 2$ & Forme & $\mathrm{KHI}$ \\
\hline eurot & 56,13 & loyer+ & 15,77 \\
salarie+ & 39,52 & condition+ & 15,77 \\
salaire+ & 39,2 & cuvre & 15,77 \\
smic & 39,2 & retraite+ & 14,66 \\
travaik & 37,84 & situation+ & 14,17 \\
augment+er & 37,33 & eleve+ & 14,17 \\
pa+yer & 35,90 & par & 14,06 \\
embauch+er & 26,47 & achat+ & 14,00 \\
scolaire+ & 26,47 & cotis+er & 14,00 \\
personne+ & 25,89 & benefici+er & 14,00 \\
argent & 25,19 & anpe & 14,00 \\
mois & 24,74 & compt+er & 13,70 \\
entreprise+ & 21,13 & gagn+er & 13,13 \\
main+ & 21,10 & seul+ & 13,03 \\
emploit & 20,07 & batiment+ & 12,44 \\
cdi & 19,32 & financ+er & 12,44 \\
allocat+ion & 17,54 & patrons & 12,44 \\
mon & 17,36 & charget & 12,40 \\
aide+ & 17,32 & retrouv+er & 12,40 \\
heure+ & 17,25 & chomeur+ & 12,23 \\
& & &
\end{tabular}

Tableau 3. Modalités des variables explicatives de la classe 1

généralement au montant de leur revenu mensuel. La classe $n^{\circ} 1$ correspond donc à des questions impliquant fortement les femmes, qu'il s'agisse de la personne qui interroge ou de la personne interrogée, et concernent avant tout des références financières et professionnelles:

Bonsoir Madame, donc vous avez fait une proposition du SMIC à 1500 euros et je voudrais savoir déjà comment vous allez le financer parce que, bon, y a beaucoup de salaires qui sont un petit peu au-dessus et donc ces salaires vont devenir, être payés au smic alors que souvent sont occupés par des gens qualifiés et donc voilà, je voulais savoir comment vous comptez financer ce, cette mise en place [...]. (Question posée par une femme à Ségolène Royal)

\section{La politique, le national et l'Europe (classe $n^{\circ} 2$ )}

La seconde classe renvoie à l'élection (présid+ent, République+, présidenti+el, candidat+, proposter, élu+) et à la gestion de l'interlocution (vous, sujet+, question+, êtes, Monsieur, votre, savoir+, avez, et les segments je vouloir, je vouloir.savoir+). D’autres thèmes apparaissent également tels que la France, l'Europe et la sécurité (France, agricult, quartier+, nationa+l, Europe, sécuritét, éducat+ion, français, européens). Cette seconde classe regroupe des 
thèmes qui dépendent du contexte électoral et de la nature de l'élection: s'agissant d'élire un nouveau président, la question politique occupe une large place dans les questions des citoyens, qu'il s'agisse des références à la campagne en cours, des orientations politiques envisagées ou des considérations nationales et extra-nationales.

Bonsoir Monsieur Bayrou, j'ai une question à vous poser sur l'Europe. Êtes-vous favorable à l'élargissement de l'Union européenne à l'Est notamment, la Turquie et l’Ukraine? (Question posée par un homme)

\begin{tabular}{l|l|l|l} 
Forme & $\mathrm{KHI} 2$ & Forme & $\mathrm{KHI} 2$ \\
\hline france & 22,09 & europe & 8,22 \\
aim+er & 21,81 & propos+er & 7,83 \\
presid+ent & 20,55 & BAYROU & 7,78 \\
vous & 18,90 & evoqu+er & 7,62 \\
polit+16 & 16,22 & concret+ & 7,36 \\
sujet+ & 13,80 & avez & 7,16 \\
agricultr & 13,65 & idee+ & 7,05 \\
republique+ & 13,61 & pays & 6,81 \\
question+ & 12,66 & elu+ & 6,73 \\
etes & 12,16 & excus+er & 6,72 \\
candidat+ & 11,20 & produit+ & 6,43 \\
MONSIEUR & 11,18 & securite+ & 6,43 \\
votre & 10,76 & parti+ & 6,43 \\
sur & 10,71 & educat+ion & 6,43 \\
quartier+ & 8,81 & comme & 5,91 \\
presidenti+el & 8,81 & francais+ & 5,87 \\
nationa+l & 8,50 & BOVE & 5,84 \\
savoir+ & 8,33 & musulman+ & 5,84 \\
service+ & 8,22 & europeen+ & 5,69 \\
revenir & 8,22 & notamment & 5,65 \\
& & &
\end{tabular}

Tableau 4. Profil lexical de la classe $n^{\circ} 2$

C'est à nouveau le sexe du candidat, mais masculin ici (cansex=m), qui est le facteur le plus corrélé avec ce lexique. On note également d'autres modalités: l'appartenance politique ( $p o l=d)$, le média $\left(m=t f_{1}\right)$ et le sexe de l'intervenant $(s e x=m)$. Il s'agit surtout de questions adressées aux candidats masculins et d'échanges homme-homme $(s 2=m m)$.

Cette classification lexicale permet de lever une possible ambigüité, liée à la double appartenance des candidats (groupe politique / groupe sexuel) : dans cette élection, les femmes étaient majoritairement situées à gauche de l'échiquier politique et les hommes à droite. Les résultats rendent plus saillante une distinction selon le sexe et invalident les autres hypothèses (impliquant le statut des formations politiques). Un calcul des spécificités 


\begin{tabular}{l|l} 
Forme & KHI2 \\
\hline${ }^{*}$ cansex=m & 50,18 \\
${ }^{*}$ pol=d & 27,36 \\
${ }^{*} m=t f 1$ & 25,07 \\
${ }^{*}$ sex $=m$ & 22,51 \\
${ }^{*}$ s2=mm & 22,35 \\
${ }^{*}$ canm=lepentf1 & 21,19 \\
${ }^{*}$ can=bové & 18,58 \\
${ }^{*}$ can=bayrou & 17,43 \\
${ }^{*}$ sexcan=mbayrou & 16,42 \\
${ }^{*}$ canm=bovétf1 & 14,87 \\
${ }^{*}$ canm=bayroutf1 & 13,52 \\
${ }^{*}$ sexcan=fbové & 10,01 \\
${ }^{*}$ canm=sarkozytf1 & 9,76 \\
${ }^{*}$ s2=fm & 9,36 \\
${ }^{*}$ sexcan=mbové & 8,22 \\
*can=villiers & 5,65
\end{tabular}

Tableau 5. Modalités des variables explicatives de la classe 2

lexicales permet de distinguer, à partir de la variable «sexe du candidat», les caractéristiques des questions adressées aux hommes de celles posées aux femmes.

\section{Des candidats présidentiables}

La première observation, sur le tableau des spécificités, concerne l'utilisation du segment président de la République (24 occurrences), qui est spécifique aux questions adressées aux hommes et significativement absent de celles posées aux femmes. Cette différence vaut également pour les formes si vous êtes élu (13 occurrences sur 14) et président (35 occurrences sur 41). Bien que les femmes convoitent la même fonction, seuls les hommes sont explicitement et fréquemment projetés dans le statut présidentiel. Cet évincement discursif est d'autant plus intéressant qu'il ne concerne pas les candidats de partis minoritaires mais plutôt l'ensemble des candidates, qu'elles représentent des partis minoritaires ou majoritaires. Cependant, la candidature de Ségolène Royal est mieux prise en compte que celle des autres candidates au regard de la distribution de la forme élue: 6 occurrences sur 9 renvoient à la candidate PS, sans que cela apparaisse pour autant comme une spécificité. Marie-George Buffet se voit même une fois interrogée sur la victoire de Ségolène Royal: «Voilà, madame Buffet bonjour, je voulais savoir si madame Ségolène Royal est élue à l'issue du deuxième tour [...] ». Sur les 22 occurrences de 
la forme élu, 21 correspondent à des questions adressées aux hommes. Audelà du statut présidentiel, les femmes sont également exclues des activités présidentielles. En effet, chargé de diriger le pays au travers d'une orientation politique et de représenter la nation sur le plan international, le futur président est censé être confronté à ces sujets. Or, ces univers de référence - gouvernance et international - distinguent nettement les hommes des femmes.

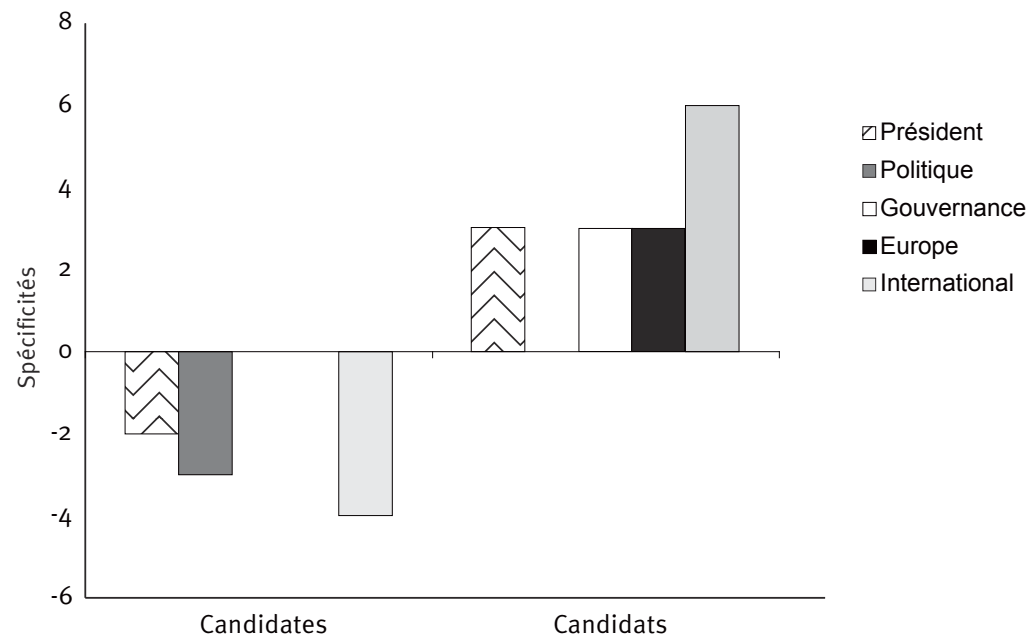

Graphique 1. Distribution de la forme président et des groupes «politique», " gouvernance», «Europe » et « international » selon le sexe des candidats

En lien avec le contexte électoral, nous avons rassemblé dans un groupe de formes ${ }^{10}$, nommé "politique», l'ensemble des références à l'univers électoral $^{11}$ : campagne, candidat, candidats, candidature, candidatures, électeur, électoral, électoraux, électeurs, élection, élections, mesure, mesures, politicienne, politiciens, politique, politiques, présidence, présidentielle, présidentielles, programme, programmes, projets, promesses, proposer, proposerez, proposez, proposition, propositions. Ce groupe de formes n'est pas spécifique aux questions posées aux hommes, mais il apparait significativement absent de celles posées aux femmes. L'accession des femmes aux hautes fonctions est d'autant moins prise en compte que la question de la gouvernance est réservée aux hommes.

10. Il s'agit de rassembler dans un même groupe un ensemble de formes lexicales choisies sur des critères linguistiques (critères morphosyntaxiques) ou extralinguistiques.

11. Le contexte des formes a été contrôlé afin de vérifier qu'elles renvoyaient bien à l'univers de référence analysé. 
Le groupe de formes "gouvernance» renvoie à l'organisation du pouvoir (assemblée, gouvernement, gouvernements, gouverner, légiférer, législation, législatives, loi, lois, ministère, ministères, ministérielle, ministériels, ministre, parlementaires). Les candidats sont non seulement projetés dans la fonction présidentielle mais également interrogés sur les pratiques du pouvoir qu'elle implique. Si ce groupe de formes n'est pas significativement absent des questions posées aux femmes, en réalité seule Ségolène Royal est interrogée sur ce sujet, étant plus à même que les autres candidates d'arriver au second tour de l'élection. Dans cette logique discriminatoire, les candidats de partis minoritaires, hommes et femmes confondus, ne sont pas considérés comme des présidentiables puisque la question de la gouvernance se concentre exclusivement autour de quatre personnalités: Nicolas Sarkozy, Jean-Marie Le Pen, Ségolène Royal et surtout François Bayrou, placé en position de «troisième homme » par les sondages.

Les hommes sont également bien plus confrontés aux questions européennes et internationales. Le groupe de formes «Europe» (Europe, européen, européenne, européennes et européens) est significativement présent dans les questions adressées aux hommes, qu'il s'agisse de références à la Constitution européenne et des suites à donner au processus et à l'organisation de l'Union européenne. De nouveau, cette thématique vise les quatre principaux présidentiables, même si pour le candidat d'extrême droite, la question européenne est abordée en partie sous l'angle de l'immigration.

Le groupe de formes «international» regroupe l'ensemble des références aux pays étrangers, européens ou non (Afrique, Allemagne, américain, américaine, anglais, Angleterre, Arafat, Blair, chiites, Chine, Françafrique, Hongrie, international, internationale, internationaux, Irak, Iran, iranienne, Irlande, Israël, israélien, israélo, Jérusalem, kurdes, Liban, mexicains, Mexique, mondial, Palestine, palestinien, Pologne, Roumanie, sunnites, Turquie, Ukraine, unis ${ }^{12}$ ). À la différence des femmes, les hommes sont souvent invités à s'exprimer sur l'international (mondialisation économique, conflits, diplomatie). José Bové et Nicolas Sarkozy sont les candidats les plus interrogés à ce sujet, alors que Marie-George Buffet, Arlette Laguiller et surtout Ségolène Royal n’y sont pas confrontées ${ }^{13}$.

Amenés à s'expliquer plus fréquemment que les femmes sur les questions politiques et sur leur vision extranationale (Europe et questions internationales), les hommes discourent donc sur leur future fonction de chef d'État dans la mesure où, bien plus que tout autre élu, le président représente son pays au niveau international.

12. La forme unis renvoie au segment États-Unis.

13. Il n'y a pas de spécificités particulières (positives ou négatives) pour les autres candidats. 


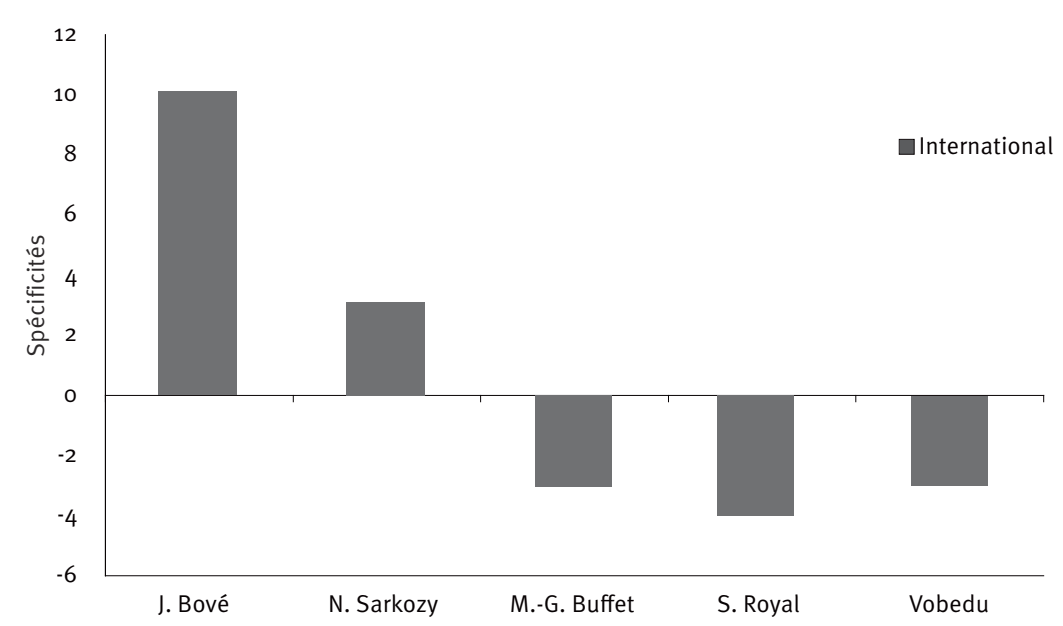

Graphique 2. Spécificités du groupe «international » selon les candidats

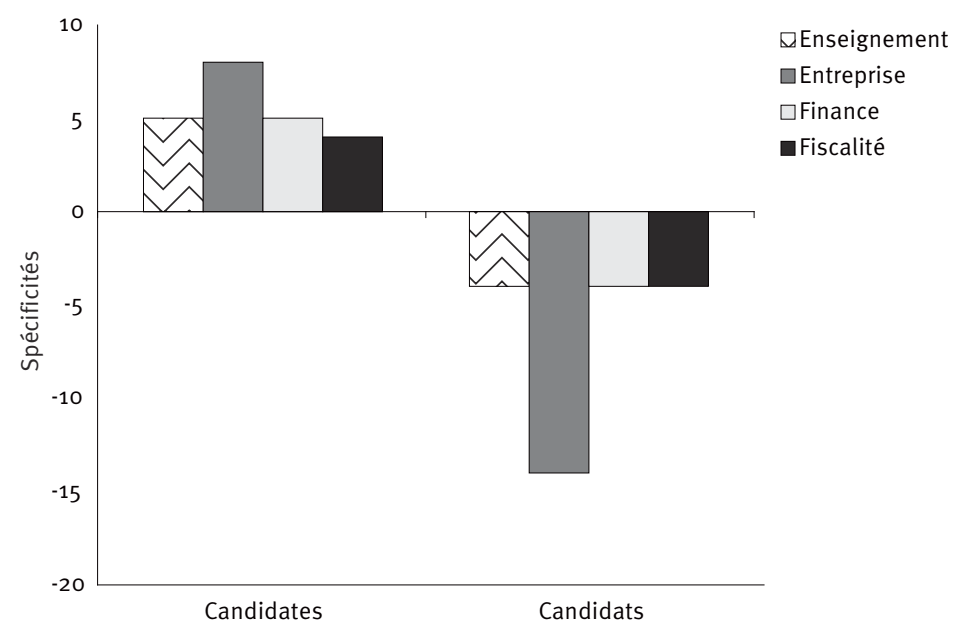

Graphique 3. Distribution des groupes «enseignement », «entreprise», «finance» et «fiscalité» selon le sexe des candidats

\section{Des candidates cantonnées à certains secteurs}

Les femmes sont invitées à s'exprimer sur trois thématiques : l'enseignement, l'entreprise et l'argent, tandis que les questions adressées aux hommes n'y font pas référence (spécificités négatives). 
Le groupe de formes «enseignement» regroupe aussi bien les lieux, les acteurs et les activités liées à l'enseignement: collège, collèges, diplômée, diplômés, école, écoles, élèves, enseignant, enseignants, enseigner, enseignement, études, étudiant, étudiants, étudier, lycée, lycéennes, lycées, prof, professeur, professeurs, rentrée, scolaire, universitaire, université, universités. Dominique Voynet mais surtout François Bayrou (7 questions) ${ }^{14}$ et Ségolène Royal ( 9 questions), qui ont tous deux occupé des fonctions ministérielles liées à l'enseignement, sont les plus interrogés sur ce sujet alors que José Bové, Jean-Marie Le Pen et Nicolas Sarkozy (2 questions) y sont moins confrontés. Outre l'enseignement, l'entreprise apparait comme l'univers référentiel qui distingue le plus les femmes des hommes. Le groupe de formes «entreprise» (chef, chefs, entrepreneur, entrepreneurs, entreprennent, entreprise, entreprises, patron, patrons, pme, salariés, sociétés, tpe) est significativement évité dans les questions posées aux hommes (MM. Bayrou, Le Pen et Sarkozy) et surtout spécifique aux candidates Arlette Laguiller et Ségolène Royal. Le thème de l'entreprise, analysé en contexte, est fortement lié à celui de la fiscalité et à l'appartenance politique des candidates: sur les huit questions uniquement consacrées à l'entreprise sur lesquelles Ségolène Royal doit s'exprimer, six concernent la lourdeur fiscale qui freine, selon les intervenants, le développement des entreprises.

Concernant le groupe de formes «finance» (finance, financée, financement, financer, financier, financièrement, financières, financiers, moyens), il s'agit surtout de questions sur la concrétisation financière des mesures prônées par les candidates: Comment financer le projet? Où trouver l'argent? Qui taxer? Ségolène Royal est la plus questionnée sur ce sujet, et ce thème se discute essentiellement entre femmes (spécificité négative pour les échanges homme-homme et pour François Bayrou) :

Bonsoir Madame Royal, donc moi j’avais une question plus générale en fait, parmi toutes vos propositions, comment vous comptez financer, qui va payer sans creuser davantage la dette? (Question posée par une femme)

En corollaire, la fiscalité (charges, cotisations, fiscal, fiscales, fiscalité, impôt, impôts, taxe, taxé, taxer, taxés, taxes) est une thématique qui touche les femmes, notamment Marie-George Buffet, et concerne essentiellement les échanges femme-femme. Les thèmes de l'entreprise et de la finance (fiscalité comprise) développés par les intervenants apparaissent comme une réaction aux mesures sociales proposées par les candidates. En ce sens, l'appartenance politique des candidates, toutes situées à gauche de l'échiquier politique, explique en partie un tel résultat. Qu'il s'agisse de la fiscalité ou de la

14. Il s'agit de questions exclusivement consacrées à cette thématique, cette dernière ayant été parfois abordée dans des questionnements plus larges. 
compatibilité entre les mesures sociales avancées et la situation financière de la France (dette de l'État...), les candidates doivent faire face aux préoccupations comptables des Français.

Mais la préoccupation pécuniaire concerne également la question du pouvoir d'achat, thème mis en avant lors de la campagne présidentielle. Si le segment pouvoir d'achat (10 occurrences) ne discrimine pas les invités politiques, il se décline par connotation au travers d'autres formes, spécifiques aux femmes: augmentation, augmenter, coût +15 , l'argent, retraites, revaloriser, smic. Les formes augmenter, charges, l'argent, retraites, smic, taxe+, 1500 sont des spécificités des questions femme-femme. L'analyse des spécificités en fonction cette fois-ci du sexe des intervenants montre que les femmes abordent plus volontiers les thématiques de l'enseignement et de l'argent. En rapport avec leur statut de mère de famille, la question de l'enseignement est fortement significative, la forme scolaire (20 occurrences) étant à elle seule uniquement abordée par les femmes (soutien scolaire, carte scolaire et allocation de rentrée scolaire). La thématique pécuniaire apparait de nouveau fortement marquée par le genre, puisque les femmes y font souvent référence. $\mathrm{Si}$ le groupe de formes «fiscalité » ne discrimine pas les intervenants, certaines formes qui le composent apparaissent en revanche spécifiques au discours féminin - c'est le cas de la forme charges ou des groupes taxe+ (taxe, taxé, taxes, taxés, taxer) et «finance».

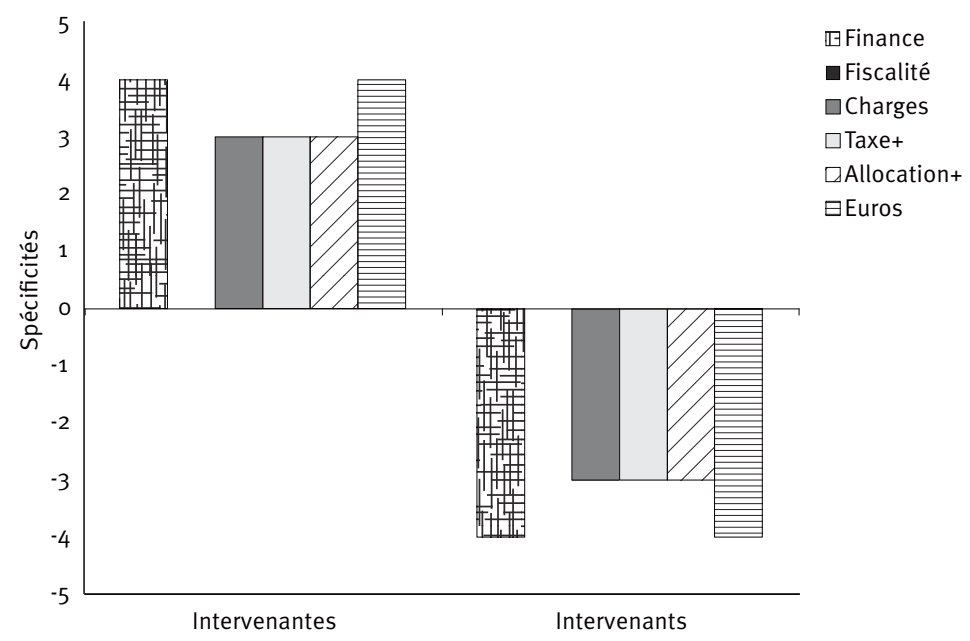

Graphique 4. Distribution des groupes «finance ", "fiscalité », «taxe+ », " allocation+ " et des formes charges et euros selon le sexe des intervenants

15. Regroupe les formes coût, coûte, coûtent, coûteuses, coûteux. 
La préoccupation pécuniaire liée au pouvoir d'achat est renforcée par les références aux allocations (allocation, allocations) et à l'emploi de la forme euros (28 occurrences féminines sur 45 au total), dans un discours chiffré mais surtout pragmatique où l'expérience individuelle et les revenus perçus sont mis en exergue:

Vous parlez de la France de demain, la France de demain elle fait partie des, elle est dans les écoles et au niveau de l'inégalité sociale des cours, au niveau des cours de soutien scolaire. Aujourd'hui, en terminale, si on a besoin de cours de soutien scolaire, il faut avoir de, des moyens, il faut avoir les moyens de pouvoir les assumer et, en fait, à 26 euros l'heure de cours en terminale, on a besoin de, si on veut deux heures de cours de maths par semaine, ça fait 50 euros par semaine, on multiplie ça par, ça va faire du 200 euros par mois, il y a une inégalité, personne ne peut, c'est pas offert à tout le monde. Que comptez-vous faire pour ça, vous parlez d'égalité des chances pour tout le monde, elle est où dans, elle est où aujourd'hui en France? [...] J'ai pas eu de réponse à ma question [...] Sur le soutien [...] Et sur les conditions de travail de la femme? (Question posée par une femme à Ségolène Royal)

Malgré le cas de Jean-Marie Le Pen, qui polarise en partie la question européenne sous l'angle de l'immigration, les références à la fonction présidentielle et à l'international (Europe comprise) ne relèvent pas de l'appartenance politique. Les deux classes mises en avant (gouvernance / diplomatie; enseignement / sphère économique) sont avant tout expliquées par les sexes respectifs des interlocuteurs: l'univers électoral et diplomatique concerne les questions adressées aux hommes alors que l'enseignement et la sphère économique (argent, fiscalité, entreprises) ${ }^{16}$ polarisent les questions posées aux femmes.

Perçues avant tout au travers de leur catégorie sexuelle, les femmes sont interrogées sur des sujets distincts de ceux des hommes et qui renvoient à des thématiques particulières. En lien avec la vision phallo-narcissique de la société (Bourdieu, 1998; Goffman, 2002) et le contexte électoral (campagne présidentielle), il s'avère que les sujets auxquels sont confrontées les candidates les discriminent dans leur accession à la fonction présidentielle. Les femmes interrogées ne sont pas considérées comme leurs pairs masculins, c'est-à-dire comme des candidats présidentiables. Conformément au postulat de Bourdieu (1998), les questions des citoyens s'avèrent ici être des questions de genre: la domination masculine ne cesse de renouveler ses formes. Ce n'est pas tant leur statut de personnalité politique qui est remis en cause, quoi qu'elles soient tout de même soumises à des représentations genrées (Derville et Pionchon, 2005; Sourd, 2005), que leur prétention à accéder à la plus haute fonction politique. Et on ne peut s'empêcher d'observer que cet effet est le plus fort lorsque le questionneur et le questionné sont du même sexe: les

16. L’économie est ici à entendre dans le sens étymologique: du grec ancien oỉkovo $\mu$ í $\alpha$ «administration d'un foyer», de oĩkoऽ «maison, dans le sens de patrimoine » et vé $\mu \omega$ «administrer». 
situations dans lesquelles, soit les femmes interrogent des femmes, soit les hommes interrogent des hommes, sont celles qui marquent le plus cette discrimination générique. Tout semble se passer comme si l'identification générique avait supplanté l'identification politique. Ce résultat confirme:

- La distinction qui peut exister entre les images que les personnalités politiques souhaitent émettre et celles qui sont construites par les citoyens. À l'inverse des hommes, les femmes doivent faire face à une discrimination sexuelle : cela implique le recours à des représentations genrées, recouvrant des normes et stéréotypes de genre encore persistants, de la part de ceux et celles qui les interrogent ${ }^{17}$.

- La prégnance du genre dans l'accession des femmes au pouvoir. Malgré leur émancipation dans les sphères publiques et politiques, les femmes subissent toujours la division sexuelle de la société (Ballmer-Cao, Mottier, Sgier, 2000 ; Achin, Lévêque, 2006). Elles demeurent encore exclues des politiques publiques traditionnellement masculines (politique étrangère, internationale, etc.) et restent cantonnées aux affaires publiques liées à l'ÉtatProvidence et au social.

\section{Références}

ACHIn Catherine, LÉVÊQUe Sandrine, 2006, Femmes en politique, Paris, La Découverte. Ballmer-Cao Thanh-Huyen, Mottier Véronique, Sgier Léa, 2000, Genre et politique. Débats et perspectives, Paris, Gallimard.

BouRdieu Pierre, 1998, La domination masculine, Paris, Le Seuil.

ChARAUdeau Patrick, 2005, Le discours politique. Les masques du pouvoir, Paris, Vuibert.

DARRAS Éric, 1994, "Un paysan à la télé. Nouvelles mises en scène du politique», Réseaux, vol. 12, nº63, p. 75-100.

DeRVILle Grégory, PIOnCHON Sylvie, 2005, "La femme invisible. Sur l'imaginaire du pouvoir politique», Mots. Les langages du politique, $\mathrm{n}^{\circ} 78$, Usages politiques $d u$ genre, p. 53-64.

Deschamps Jean-Claude, Moliner Pascal, 2008, L'identité en psychologie sociale. Des processus identitaires aux représentations sociales, Paris, Armand Colin.

GoffMAn Erving, 2002, L'arrangement des sexes, Paris, La Dispute.

Lebart Ludovic, Salem André, 1994, Statistique textuelle, Paris, Dunod.

LE Grignou Brigitte, NeVEu Érik, 1997, «Émettre la réception. Préméditations et réceptions de la politique télévisée», Sociologie de la communication, Réseaux Reader CNET, P. Beaud, P. Flichy, D. Pasquier, L. Quéré éd., p. 749-771.

17. La volonté de Ségolène Royal d'afficher une différence fondée sur le sexe durant la précampagne et la campagne pourrait être invoquée pour expliquer les présents résultats. Mais elle ne permet pas d'expliquer que les questions des citoyens discriminent d'un côté l'ensemble des hommes et de l'autre, les femmes: les présents résultats ne concernent pas des individus considérés isolément. 
Marchand Pascal, 2004, Psychologie sociale des médias, Rennes, Presses universitaires de Rennes.

Marchand Pascal, Pino Michaël, 2007, «Dis-moi quels médias tu préfères, je te dirai comment tu votes », Communication (Canada), $n^{\circ} 25$ (2), p. 36-78.

McCOMBS Maxwell, REYNOLDS Amy, 2002, «News influence on our pictures of the world», Media effects, J. Bryant, D. Zillmann éd., New Jersey, Lawrence Erlbaum, p. 1-18.

MOLINER Pascal, 1996, Images et représentations sociales. De la théorie des représentations à l'étude des images sociales, Grenoble, Presses universitaires de Grenoble.

Mouchon Jean, 1995, "La communication présidentielle en quête de modèles », Hermès, $n^{\circ} 17-18$, p. 187-200.

- 2001, "Le résistible déclin du débat public à la télévision, Mots. Les langages du politique, $\mathrm{n}^{\circ} 67$.

NADEAU Richard, GIASSON Thierry, 2003, «Les médias et le malaise démocratique au Canada», Choix, n 9 (1), p. 1-32.

NEVEU Érik, 1995, «Les émissions politiques à la télévision. Les années quatre-vingt ou les impasses du spectacle politique », Hermès, $\mathrm{n}^{\circ} 17-18$, p. 145-162.

REINERTMax, 1990, «Alceste, une méthodologie d'analyse des données textuelles et une application : Aurélia de Gérard de Nerval », Bulletin de méthodologie sociologique, $\mathrm{n}^{\circ} 26$, p. 24-54.

SouRd Cécile, 2005, «Femmes ou politiques? La représentation des candidates aux éléctions françaises de 2002 dans la presse hebdomadaire», Mots. Les langages du politique, $\mathrm{n}^{\circ} 78$, Usages politiques du genre, p. 65-77. 